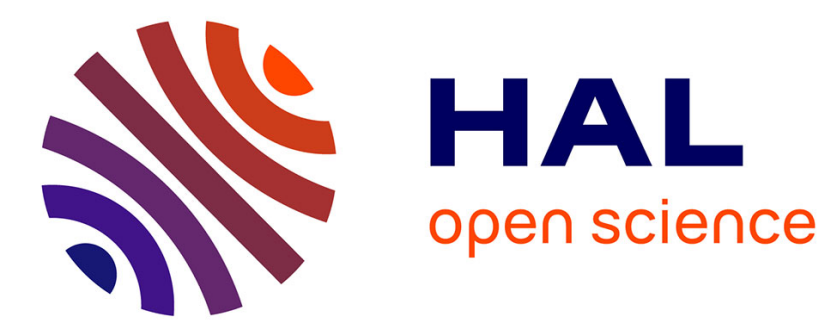

\title{
Probing optomechanical correlations between two optical beams down to the quantum level
}

P. Verlot, A. Tavernarakis, T. Briant, P. -F. Cohadon, A. Heidmann

\section{To cite this version:}

P. Verlot, A. Tavernarakis, T. Briant, P. -F. Cohadon, A. Heidmann. Probing optomechanical correlations between two optical beams down to the quantum level. Physical Review Letters, 2009, 102, pp.103601. 10.1103/PhysRevLett.102.103601 . hal-00321609

\section{HAL Id: hal-00321609 \\ https://hal.science/hal-00321609}

Submitted on 15 Sep 2008

HAL is a multi-disciplinary open access archive for the deposit and dissemination of scientific research documents, whether they are published or not. The documents may come from teaching and research institutions in France or abroad, or from public or private research centers.
L'archive ouverte pluridisciplinaire HAL, est destinée au dépôt et à la diffusion de documents scientifiques de niveau recherche, publiés ou non, émanant des établissements d'enseignement et de recherche français ou étrangers, des laboratoires publics ou privés. 


\title{
Probing optomechanical correlations between two optical beams down to the quantum level
}

\author{
P. Verlot, A. Tavernarakis, T. Briant, P.-F. Cohadon, and A. Heidmann \\ Laboratoire Kastler Brossel, UPMC-ENS-CNRS, Case 74, \\ 4 place Jussieu, F75252 Paris Cedex 05, France
}

(Dated: September 15, 2008)

\begin{abstract}
Quantum effects of radiation pressure are expected to limit the sensitivity of second-generation gravitational-wave interferometers. Though ubiquitous, such effects are so weak that they haven't been experimentally demonstrated yet. Using a high-finesse optical cavity and a classical intensity noise, we have demonstrated radiation-pressure induced correlations between two optical beams sent into the same moving mirror cavity. Our scheme can be extended down to the quantum level and has applications both in high-sensitivity measurements and in quantum optics.
\end{abstract}

PACS numbers: 42.50.Wk, 05.40.Jc, 03.65.Ta

Quantum effects of optomechanical coupling, the radiation-pressure coupling between a moving mirror and an incident light field, were first studied in the framework of gravitational-wave detection [1, 2, 3], enforcing quantum limits to the sensitivity of large-scale interferometers [4, 5, 5. Overcoming these limits was a major motivation for the quantum optics experiments performed shortly after, such as squeezing of the light field [7, 81 or quantum non demolition (QND) measurements 9 , 10, 11. Such pioneering experiments were performed with nonlinear optical media, but optomechanical coupling was soon proposed as a candidate nonlinear mechanism of its own [12, 13, 14, based upon correlations between light intensity and mirror displacement induced by radiation pressure.

The first experiments fell short of the quantum regime [15, 16, 17] and even though recent ones demonstrated a much larger optomechanical coupling [18, 19, 20, 21, 22, 23], they mainly focussed on the possible demonstration of the quantum ground state of a mechanical resonator [24, 25]. To observe the optomechanical correlations, two beams have to be sent upon the moving mirror (see Fig. 1): the intensity fluctuations of the first, intense, signal beam drive the mirror into motion by radiation pressure, whereas the resulting position fluctuations are monitored through the phase of the second, weaker, meter beam. As the intensity fluctuations of the signal beam are unaltered by reflection upon the mirror and as far as the radiation pressure of the meter beam is negligible, the intensity-phase correlations observable between the two reflected beams provide a direct measurement of the optomechanical correlations.

To monitor these radiation-pressure effects down to the quantum level and hence perform a real-time QND measurement of the signal intensity via the meter phase [14, one has first to enhance the optomechanical coupling by using a high-finesse cavity with a moving mirror, as shown in Fig. 1. The position fluctuations $\delta x_{\text {rad }}$ induced by the quantum intensity fluctuations of the signal beam also have to be the dominant noise source, which requires to lower the thermal fluctuations $\delta x_{\mathrm{T}}$ of the moving mirror. For a harmonic oscillator of mass $M$, resonance

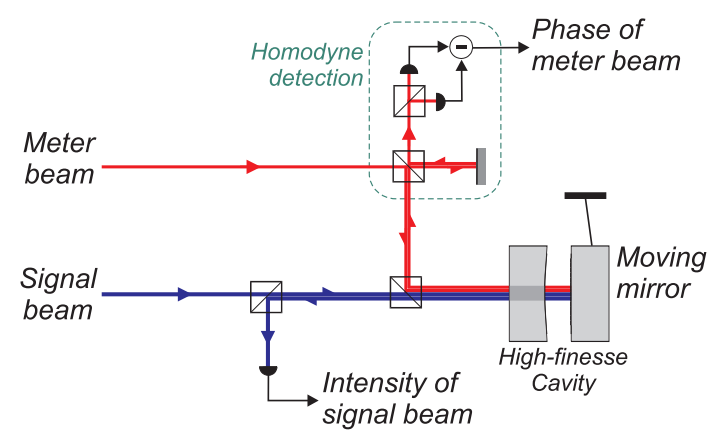

FIG. 1: Principle of the direct observation of optomechanical correlations. Both an intense signal beam and a weaker meter beam are sent into a resonant high-finesse cavity with a moving mirror. Intensity fluctuations of the signal beam are imprinted by radiation pressure onto the position fluctuations of the moving mirror, and subsequently onto the phase fluctuations of the meter beam. The two reflected beams then display intensity-phase correlations, retrieved with both a photodiode and a homodyne detection.

frequency $\Omega_{\mathrm{M}} / 2 \pi$, and mechanical quality factor $Q$, the corresponding ratio between the radiation pressure and thermal noise spectra can be written 14.

$$
\begin{aligned}
\frac{S_{x}^{\mathrm{rad}}}{S_{x}^{\mathrm{T}}} \simeq & 2.3\left(\frac{\mathcal{F}}{300000}\right)^{2}\left(\frac{800 \mathrm{~nm}}{\lambda}\right)\left(\frac{P_{\text {in }}}{1 \mathrm{~mW}}\right) \\
& \times\left(\frac{1 \mathrm{mg}}{M}\right)\left(\frac{Q}{10^{6}}\right)\left(\frac{1 \mathrm{MHz}}{\Omega_{\mathrm{M}} / 2 \pi}\right)\left(\frac{1 \mathrm{~K}}{T}\right)
\end{aligned}
$$

where $T$ is the environment temperature, $\mathcal{F}$ the cavity finesse, $\lambda$ the optical wavelength, and $P_{\text {in }}$ the incident intensity of the signal beam. The stated values have all already been achieved independently in various state-ofthe-art optomechanical systems [18, 19, 20, 21, 22, 23, 26, 27, but combining the favourable mechanical behaviour of NEMS [26] with a very high optical finesse [27] is an even greater experimental challenge.

In this work, we report the observation of optomechanical correlations measured close to the quantum level. To reach a ratio (1) as large as possible, we favour the optical characteristics and use a fused silica moving mirror, 


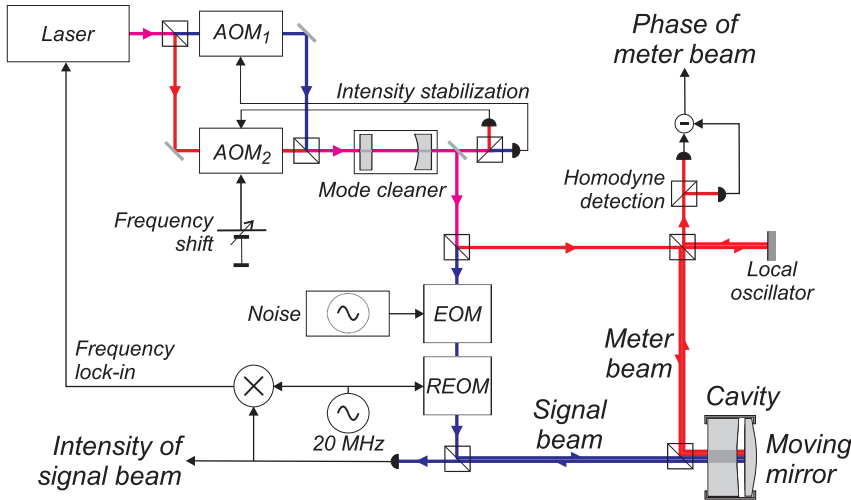

FIG. 2: Experimental setup. The laser beam is split in two orthogonally polarized beams, which are both sent into the moving mirror cavity. A resonant electro-optical modulator (REOM) is used to lock the laser onto the optical resonance via a Pound-Drever-Hall technique. The residual birefringence of the cavity is compensated by the frequency shift of two acousto-optic modulators (AOM), also used to stabilize the intensities of both beams after their spatial filtering by the mode cleaner cavity. A second EOM modulates the intensity of the signal beam to mimic quantum radiation-pressure noise. Intensity fluctuations of the reflected signal beam are monitored with a photodiode, as are the phase fluctuations of the meter beam with a quantum-limited homodyne detection. For simplicity, most polarizing elements are not shown.

which provides both a very high optical finesse [27] and mechanical quality factor [28], at the expense of a larger mass. The optomechanical correlations have then been measured with a tiny classical intensity modulation of the signal beam that mimics at a higher level its quantum fluctuations 27, 29].

Our experimental setup is based on a single-ended optical cavity, with a 1-inch fused silica cylindrical input mirror. The moving mirror, used as end mirror, is a plano-convex $34-\mathrm{mm}$ diameter and 2.5 - $\mathrm{mm}$ thick mirror, which displays gaussian internal vibration modes 28 . We work at frequencies close to a mechanical resonance with the following optomechanical characteristics, deduced from the thermal noise spectrum at room temperature: $\Omega_{\mathrm{M}} / 2 \pi=1.125 \mathrm{MHz}, M=500 \mathrm{mg}, Q=500000$.

The low roughness of the silica substrates allows for optical coatings with very low losses: we have obtained a cavity finesse $\mathcal{F}=330000$, mainly limited by the 20 -ppm transmission of the input mirror. This is crucial for quantum optics experiments for which loss has to be avoided to get large correlations between intracavity and reflected fields. We use a short, 0.33-mm long, cavity in order to keep a sufficient cavity bandwidth $\left(\Omega_{\text {cav }} / 2 \pi=700 \mathrm{kHz}\right)$ and to prevent laser frequency noise from limiting the displacement sensitivity. The cavity is operated in vacuum to increase the mechanical quality factors.

The cross-polarized signal and meter beams entering the cavity are provided by a Ti:Sa laser working at 810 $\mathrm{nm}$. As the cavity is birefringent (with a $5-\mathrm{MHz}$ frequency mismatch between the two optical resonances), two acousto-optic modulators (AOM in Fig. 2) independently detune the two beams so that they both match the cavity resonance. The overall resonance is controlled by locking the laser frequency via a PoundDrever-Hall technique: the incident signal beam is phasemodulated at $20 \mathrm{MHz}$ by a resonant electro-optical modulator (REOM), and the resulting intensity modulation of the reflected beam provides the error signal. A mode cleaner cavity filters potential degradations of the spatial profile of both beams, while their intensities after the mode cleaner are stabilized by a servo-loop which drives the amplitude control of the AOMs.

The phase fluctuations $\delta \varphi_{m}^{\text {out }}(t)$ of the reflected meter beam are monitored by a homodyne detection, with a local oscillator derived from the incident meter beam and phase-locked in order to detect the phase quadrature. For an incident power of $50 \mu \mathrm{W}$, one gets a shot-noise-limited displacement sensitivity of $2.7 \times 10^{-20} \mathrm{~m} / \sqrt{\mathrm{Hz}}$ at frequencies above $200 \mathrm{kHz}$. Intensity fluctuations $\delta I_{s}^{\text {out }}(t)$ of the reflected signal beam are monitored by a high-efficiency photodiode. We have carefully eliminated unwanted optical reflections so that the optical rejection of the doublebeam system is higher than $35 \mathrm{~dB}$ : the phase fluctuations of the meter beam are insulated from the intensity fluctuations of the signal beam in such a way that observable effects of the signal beam are necessarily induced by intracavity radiation pressure.

In order to mimic the quantum fluctuations of radiation pressure, the signal beam is intensity-modulated with an electro-optic modulator (EOM) before entering the cavity to produce a classical intracavity radiationpressure noise [27, 29]. The digitized driving noise is centered at a frequency $\Omega_{\mathrm{c}}$ close to the mechanical resonance frequency $\Omega_{\mathrm{M}}$, and has a typical bandwidth of a few hundreds of $\mathrm{Hz}$, larger than any bandwidth used in the correlations acquisition process. To generate a gaussian intensity noise of the form $\delta I_{s}^{\text {in }}(t)=A(t) \cos \left(\Omega_{\mathrm{c}} t+\varphi(t)\right)$ where $A(t)$ is a random function with a gaussian distribution around 0 and $\varphi(t)$ a randomly-distributed phase, we decompose the noise into its quadratures 30]:

$$
\delta I_{s}^{\mathrm{in}}(t)=X_{I_{s}}^{\mathrm{in}}(t) \cos \left(\Omega_{\mathrm{c}} t\right)+Y_{I_{s}}^{\mathrm{in}}(t) \sin \left(\Omega_{\mathrm{c}} t\right) .
$$

The quadratures are produced from a dual-channel arbitrary waveform generator Tektronix AFG3022B, and then summed to drive the EOM. The slowly-varying gaussian noise functions $X_{I_{s}}^{\text {in }}(t)$ and $Y_{I_{s}}^{\text {in }}(t)$ are randomly generated by a computer and loaded into the generator as amplitude arrays.

The experiment is performed as follows. Both optical beams are locked onto the resonance of the cavity, with incident powers $P_{s}^{\text {in }}=150 \mu \mathrm{W}$ for the signal beam and $P_{m}^{\text {in }}=500 \mu \mathrm{W}$ for the meter. The EOM drives a classical radiation-pressure noise with an amplitude level as compared to thermal noise of $\sqrt{S_{x}^{\mathrm{rad}} / S_{x}^{\mathrm{T}}} \simeq 5$, and with a center frequency $\Omega_{\mathrm{c}} / 2 \pi=1.123 \mathrm{MHz}$, about 600 mechanical linewidths below the mechanical resonance. The experimental signals are independently acquired by two spectrum analyzers Agilent MXA set in I/Q mode in or- 


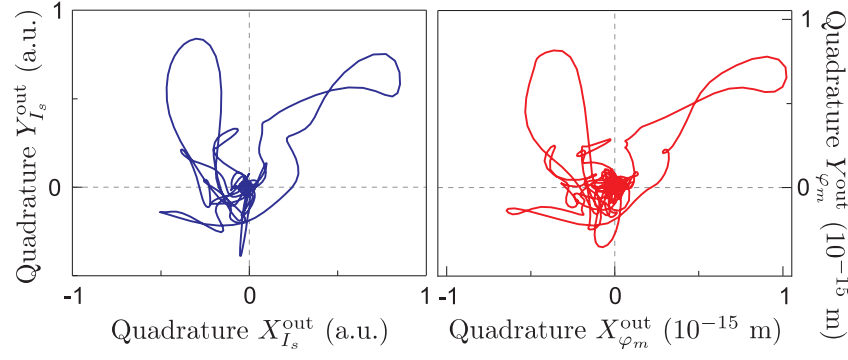

FIG. 3: Phase-space trajectories of the intensity noise of the signal beam (left) and the phase noise of the meter beam (right), in the case $\sqrt{S_{x}^{\mathrm{rad}} / S_{x}^{\mathrm{T}}} \simeq 5$. The phase noise is calibrated as displacements of the moving mirror.

der to directly extract the quadratures $X_{I_{s}}^{\text {out }}(t), Y_{I_{s}}^{\text {out }}(t)$, $X_{\varphi_{m}}^{\text {out }}(t)$, and $Y_{\varphi_{m}}^{\text {out }}(t)$ of the reflected signal intensity and meter phase, respectively. Both analyzers are locked at the same central frequency $\Omega_{\mathrm{c}}$ with an analysis bandwith of $400 \mathrm{~Hz}$, and synchronously triggered with the waveform generator. Temporal evolution of the quadratures are then acquired over a span time of $200 \mathrm{~ms}$, equal to the scan time of the digitized amplitude-modulation arrays of the generator.

Fig. 3 presents the observed phase-space trajectories: clear correlations are evident between the intensity noise of the signal beam (left) and the meter phase noise (right). Neglecting optical losses and irrelevant noises such as the quantum fluctuations of the meter beam, this can be interpreted from the following input-output relations for the fluctuations at frequency $\Omega_{\mathrm{c}}$ [14]:

$$
\begin{aligned}
\delta I_{s}^{\text {out }}\left[\Omega_{\mathrm{c}}\right] & =\frac{1+i \omega_{\mathrm{c}}}{1-i \omega_{\mathrm{c}}} \delta I_{s}^{\mathrm{in}}\left[\Omega_{\mathrm{c}}\right], \\
\delta \varphi_{m}^{\text {out }}\left[\Omega_{\mathrm{c}}\right] & =\frac{8 \mathcal{F}}{\lambda\left(1-i \omega_{\mathrm{c}}\right)} \delta x\left[\Omega_{\mathrm{c}}\right],
\end{aligned}
$$

where $\omega_{\mathrm{c}}=\Omega_{\mathrm{c}} / \Omega_{\mathrm{cav}}$, and $\delta x=\delta x_{\mathrm{T}}+\delta x_{\mathrm{rad}}$ is the mirror motion, including the thermal noise and the radiationpressure noise given by

$$
\delta x_{\mathrm{rad}}\left[\Omega_{\mathrm{c}}\right]=\frac{8 \mathcal{F}}{\lambda\left(1-i \omega_{\mathrm{c}}\right)} \hbar \chi\left[\Omega_{\mathrm{c}}\right] \delta I_{s}^{\mathrm{in}}\left[\Omega_{\mathrm{c}}\right],
$$

where $\chi\left[\Omega_{\mathrm{c}}\right]$ is the mechanical susceptibility of the moving mirror. The reflected signal intensity noise reproduces the incident one, with a global phase shift depending on $\omega_{c}$ [eq. (3)], whereas the reflected meter phase reproduces the incident signal intensity $\delta I_{s}^{\text {in }}$ via the mirror motion [eqs. (4) and (5)]. It is superimposed to the thermal noise $\delta x_{\mathrm{T}}$ of the mirror which is responsible for the small differences observed between the two phase-space evolutions in Fig. 3. Other noises such as the quantum phase noise of the incident meter beam, which limits the sensitivity of the displacement measurement, are negligible in our current setup with a level at least $15 \mathrm{~dB}$ below the thermal noise. Also note that the meter phase in Fig. 3 is calibrated in terms of the equivalent displacements

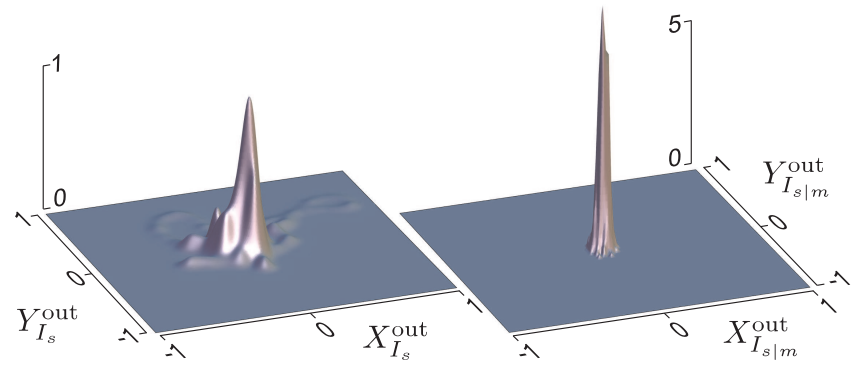

FIG. 4: Probability distributions in phase space of the signal intensity fluctuations $\delta I_{s}^{\text {out }}$ (left) and of the conditional fluctuations $\delta I_{s \mid m}$ deduced from the meter measurement (right). Note the sharper peak, related to the lower conditional variance, and the factor 5 between the two vertical scales.

of the moving mirror, with a typical level at $10^{-15} \mathrm{~m}$, and the curve has been rotated in phase space in order to compensate for the global phase shifts due to $\omega_{c}$ and to the mechanical response $\chi\left[\Omega_{\mathrm{c}}\right]$.

We have obtained similar results with a center frequency $\Omega_{\mathrm{c}}$ closer or equal to the mechanical resonance frequency. In that case, the resonance amplifies the radiation-pressure and thermal displacements by a factor up to the quality factor $Q$, but the phase shift of the mechanical response across the resonance frequency has to be taken into account to deconvolve the observed data. We focus in the following on experimental results obtained at low frequency.

The results can be made more quantitative by computing the correlation coefficient $C_{I_{s}, \varphi_{m}}$ defined from the two trajectories in phase-space:

$$
C_{I_{s}, \varphi_{m}}=\frac{\mid\left\langle\left.\delta I_{s}^{\text {out }} \delta \varphi_{m}^{\text {out } \left.^{\star}\right\rangle}\right|^{2}\right.}{\left\langle\left|\delta I_{s}^{\text {out }}\right|^{2}\right\rangle\left\langle\left\langle\left.\delta \varphi_{m}^{\text {out }}\right|^{2}\right\rangle\right.}
$$

where the brackets $\langle\ldots\rangle$ stand for a temporal average. We obtain a coefficient $C_{I_{s}, \varphi_{m}} \simeq 0.96$ for the data presented on Fig. 3, in perfect agreement with the value $\left(1+S_{x}^{\mathrm{T}} / S_{x}^{\mathrm{rad}}\right)^{-1}$ deduced from Eqs. (3) to (5).

As in usual QND measurements [9], optomechanical correlations can also be quantified by the knowledge we have on the signal intensity from the measurement of the meter phase. The resulting distribution is given by the conditional fluctuations

$$
\delta I_{s \mid m}=\delta I_{s}^{\text {out }}-\frac{\left\langle\delta I_{s}^{\text {out }} \delta \varphi_{m}^{\text {out }}\right\rangle}{\left\langle\left|\delta \varphi_{m}^{\text {out }}\right|^{2}\right\rangle} \delta \varphi_{m}^{\text {out }} .
$$

Figure 1 presents the respective probability distributions in phase space for the uncorrected intensity fluctuations $\delta I_{s}^{\text {out }}$ and the conditional ones $\delta I_{s \mid m}$, obtained as normalized histograms of the data of Fig. 3. The shrinking of the distribution is related to the lower conditional dispersion, reduced by a factor $\simeq 5$, as can be deduced from Eqs. (3) to (7):

$$
\Delta I_{s \mid m}=\sqrt{1-C_{I_{s}, \varphi_{m}}} \Delta I_{s}^{\text {out }} \simeq 0.2 \Delta I_{s}^{\text {out }} .
$$




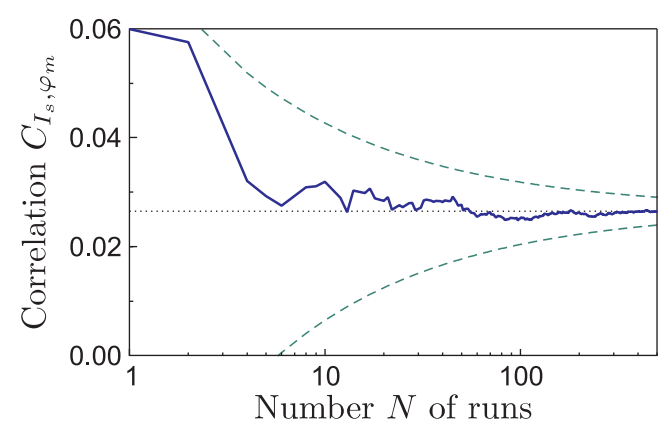

FIG. 5: Estimated optomechanical correlation coefficient $C_{I_{s}, \varphi_{m}}$ with respect to the number $N$ of independent 200-ms runs averaged. Dashed lines delineate the expected $1 / \sqrt{N}$ statistical uncertainty region.

Our experimental setup enables to demonstrate optomechanical correlations even in the case of radiation-pressure effects smaller than the thermal noise $\left(S_{x}^{\mathrm{rad}} \ll S_{x}^{\mathrm{T}}\right)$. In such a case, as the reflected meter phase fluctuations $\delta \varphi_{m}^{\text {out }}$ are mainly related to random thermal noise, the correlation coefficient deduced from the temporal average of a single 200-ms run has little meaning, and experimental values fluctuate from one run to the other. Nevertheless, repeating such runs and averaging all these experimental outcomes eventually yields a steady value.

Fig. 5 presents the estimate of the correlation coefficient obtained with $S_{x}^{\mathrm{rad}} / S_{x}^{\mathrm{T}} \simeq 0.03$, as a function of the number $N$ of runs averaged, up to $N=500$. The resulting correlation coefficient tends to its small but non-zero expected value $\left(1+S_{x}^{\mathrm{T}} / S_{x}^{\mathrm{rad}}\right)^{-1} \simeq 0.03$, with a statistical uncertainty at least 10 -times smaller $\left(2.5 \times 10^{-3}\right.$ for 500 averages).

We have thus demonstrated optomechanical correlations between two light beams. Such correlations are still at the classical level but we note that for our system, $S_{x}^{\mathrm{rad}} / S_{x}^{\mathrm{T}} \simeq 10^{-3}$ for quantum noise and a temperature of $1 \mathrm{~K}$. Averaging the experimental signal once working at low temperature should enable to retrieve the corresponding quantum correlations and hence demonstrate radiation-pressure noise, which is expected to be a severe limitation of second-generation gravitational-wave interferometers [6]. With an upgrade of our experimental setup, one can also envision radiation-pressure induced quantum optics experiments, such as optomechanical squeezing [12] or QND measurements [13, 14].

We gratefully acknowledge the Laboratoire des Matériaux Avancés for the low-loss optical coating of the mirrors. We are grateful to Matteo Barsuglia and Eric Chassande-Mottin for fruitful discussions. This work was partially funded by EGO (collaboration convention EGO-DIR-150/2003 for a study of quantum noises in gravitational wave interferometers) and by the Integrated Large Infrastructures for Astroparticle Science (ILIAS) of the Sixth Framework Program of the European Community.
[1] V.B. Braginsky and F. Ya Khalili, Quantum Measurement (Cambridge University Press, 1992).

[2] C. Bradaschia et al., Nucl. Instrum. Meth. A 289, 518 (1990).

[3] A. Abramovici et al., Science 256, 325 (1992).

[4] C.M. Caves, Phys. Rev. D 23, 1693 (1981).

[5] M.T. Jaekel and S. Reynaud, Europhys. Lett. 13, 301 (1990).

[6] P. Fritschel, Proc. SPIE 4856, 282 (2003).

[7] D.F. Walls, Nature (London) 306, 141 (1983).

[8] R.E. Slusher, L.W. Hollberg, B. Yurke, J.C. Mertz, and J.F. Valley, Phys. Rev. Lett. 55, 2409 (1985).

[9] P. Grangier, J.A. Levenson, J.-P. Poizat, Nature (London) 396, 537 (1998).

[10] N. Imoto, H.A. Haus, and Y. Yamamoto, Phys. Rev. A 32, 2287 (1985)

[11] J.-F. Roch et al., Phys. Rev. Lett. 78, 634 (1997).

[12] C. Fabre et al., Phys. Rev. A 49, 1337 (1994).

[13] K. Jacobs, P. Tombesi, M.J. Collett, and D.F. Walls, Phys. Rev. A 49, 1961 (1994).

[14] A. Heidmann, Y. Hadjar, and M. Pinard, Appl. Phys. B 64, 173 (1997).

[15] Y. Hadjar, P.-F. Cohadon, C.G. Aminoff, M. Pinard, and A. Heidmann, Europhys. Lett. 47, 545 (1999).

[16] P.-F. Cohadon, A. Heidmann, and M. Pinard, Phys. Rev. Lett. 83, 3174 (1999).
[17] I. Tittonen et al., Phys. Rev. A 59, 1038 (1999).

[18] O. Arcizet et al., Phys. Rev. Lett. 97, 133601 (2006).

[19] O. Arcizet, P.-F. Cohadon, T. Briant, M. Pinard, and A. Heidmann, Nature (London) 444, 71 (2006).

[20] S. Gigan et al., Nature (London) 444, 67 (2006).

[21] T. Corbitt et al., Phys. Rev. Lett. 99, 160801 (2007).

[22] J.D. Thompson et al., Nature (London) 452, 72 (2008).

[23] A. Schliesser, O. Rivière, G. Anetsberger, O. Arcizet, and T.J. Kippenberg, Nature Physics 4, 415 (2008).

[24] R.G. Knobel and A.N. Cleland, Nature (London) 424, 291 (2003).

[25] M.D. LaHaye, O. Buu, B. Camarota, and K.C. Schwab, Science 304, 74 (2004).

[26] D. Rugar, R. Budakian, H.J. Mamin, and B.W. Chui, Nature (London) 430, 329 (2004).

[27] T. Caniard, P. Verlot, T. Briant, P.-F. Cohadon, and A. Heidmann, Phys. Rev. Lett. 99, 110801 (2007).

[28] T. Briant, P.-F. Cohadon, A. Heidmann, and M. Pinard, Phys. Rev. A 68, 033823 (2003).

[29] C.M. Mow-Lowry, B.S. Sheard, M.B. Gray, D.E. McClelland, and S.E. Whitcomb, Phys. Rev. Lett. 92, 161102 (2004).

[30] T. Briant, P.-F. Cohadon, M. Pinard, and A. Heidmann, Eur. Phys. J. D 22, 131 (2003). 